\title{
Nanoscale swimmers: hydrodynamic interactions and propulsion of molecular machines
}

\author{
T. Sakaue ${ }^{1, a}$, R. Kapral ${ }^{2}$, and A.S. Mikhailov ${ }^{3}$ \\ 1 Department of Physics, Kyushu University 33, 812-8581 Fukuoka, Japan \\ and \\ PRESTO, Japan Science and Technology Agency (JST), 4-1-8 Honcho Kawaguchi, Saitama 332-0012, Japan \\ 2 Chemical Physics Theory Group, Department of Chemistry, University of Toronto, Toronto, Ontario, M5S 3H6 Canada \\ 3 Abteilung Physikalische Chemie, Fritz-Haber-Institut der Max-Planck-Gesellschaft, Faradayweg 4-6, 14195 Berlin, Germany
}

Received 25 June 2009 / Received in final form 19 November 2009

Published online 6 May 2010 - (c) EDP Sciences, Società Italiana di Fisica, Springer-Verlag 2010

\begin{abstract}
Molecular machines execute nearly regular cyclic conformational changes as a result of ligand binding and product release. This cyclic conformational dynamics is generally non-reciprocal so that under time reversal a different sequence of machine conformations is visited. Since such changes occur in a solvent, coupling to solvent hydrodynamic modes will generally result in self-propulsion of the molecular machine. These effects are investigated for a class of coarse grained models of protein machines consisting of a set of beads interacting through pair-wise additive potentials. Hydrodynamic effects are incorporated through a configuration-dependent mobility tensor, and expressions for the propulsion linear and angular velocities, as well as the stall force, are obtained. In the limit where conformational changes are small so that linear response theory is applicable, it is shown that propulsion is exponentially small; thus, propulsion is nonlinear phenomenon. The results are illustrated by computations on a simple model molecular machine.
\end{abstract}

\section{Introduction}

Molecular machines, functioning as motors, ion pumps or enzymes, play important roles in biological cells [1,2]. Similar to macroscopic machines, their operation involves internal mechanical motions which, however, take place within a single protein molecule. Such conformational motions are robustly repeated in each succeeding operation cycle. In motors such as myosin or kinesin, they are used to move the molecule with its attached cargo along a filament $[3,4]$ (to transform cyclic shape changes of proteins into directed translational or rotational movements, ratchets or other mechanisms can be employed). In machine-like enzymes, such as phosphoglycerate kinase or adenylate kinase, functional conformational motions can facilitate catalytic reaction events by bringing the substrate-enzyme complex to an optimal configuration. Molecular machines typically receive the energy needed for their operation in chemical form through binding of a ligand, e.g. an ATP molecule. Understanding the physical mechanisms of operation of molecular machines and designing similar artificial nanoscale devices $[5,6]$ are major scientific challenges.

In his well-known study [7], Purcell analyzed the hydrodynamical propulsion of microorganisms in the low Reynolds number regime. He showed that an object cyclically changing its shape may propel itself through the fluid, i.e. it can swim [8]. The necessary condition for self-propulsion is that, within a cycle, the forward and

\footnotetext{
${ }^{a}$ e-mail: sakaue@phys.kyushu-u.ac.jp
}

backward conformational motions do not coincide so that time-reversal symmetry is broken. Propulsion phenomena have been quantitatively analyzed for a few systems, such as the three-rod swimmer $[7,9,10]$, the swimmer consisting of three linked beads [11-15] and the butterfly swimmer [16]. Recently, an extension to the $N$ sphere model has also been presented [17]. In these studies, motions of each element are assumed to be known and using this information, the propulsion velocity of a swimmer is calculated.

Molecular machines operate in a condensed phase environment and their cyclic conformational motions are also accompanied by hydrodynamical flows. Purcell's analysis in terms of asymmetrical cyclic shape changes holds as well for much smaller single-molecule-machine swimmers. The operation cycles of such machines are usually not symmetric under time-reversal. Therefore, molecular machines should generally swim if they are freely immersed in solution, and generate a propulsion force if they are bound to a membrane or other support. Such swimming motions and related stall forces are common properties of various molecular machines, from enzymes and ion pumps to motor proteins. Note that, in the case of molecular motors, hydrodynamic propulsion forces act in addition to the stronger molecular forces responsible for the ordered translational motion of the protein (with an attached cargo) along a filament.

The aims of this study are to investigate the propulsion properties of molecular machines and provide analytical expressions for their propulsion velocities and the 
stall forces needed to immobilize them. To this end, we notice that the internal motions of molecular machine are not prescribed, but rather determined by the dynamics in the associated energy landscape. Therefore, we will introduce the model in which, instead of internal motions themselves, internal forces acting between elements depending on their relative positions are given. These forces induce both the internal motions and the propulsion of the entire body.

In the next section, a generic model of a molecular machine is considered. A protein machine is modelled as a set of particles (beads), representing coarse grained molecular groups, with pair-wise potential energy interactions among them. Binding of an energy-bringing ligand, such as an ATP molecule, is described as a structural perturbation modifying the form of the free energy of the biopolymer molecule. After such a structural perturbation, the machine undergoes conformational relaxation to the new conformational state. When the ligand has been converted into the product and the product has left the protein, the machine undergoes the reverse relaxation process to its initial equilibrium state. The machine is immersed in solution and, therefore, conformational relaxation processes are accompanied by hydrodynamical flows. The effects of such flows on conformational dynamics are taken into account by a conformation-dependent mobility tensor. In Section 2, expressions for the propulsional translation and angular velocities are given for an arbitrary machine configuration. They allow one to compute such velocities when the forward and reverse conformational motions inside a cycle are known. Expressions for the stall force needed to prevent translational motion by immobilizing one of the beads are also presented in this section. Molecular machines with small deformation cycles are considered in Section 4. In such machines, the effects of energy-bringing ligands are weak and can be described within the linear response approximation. We show that propulsion vanishes within the linear approximation and, thus, the propulsion velocity and the stall force are nonlinear effects. In Section 5, the results are illustrated by calculations on a small molecular machine comprising four beads. The paper ends with conclusions and a discussion of the results.

\section{Hydrodynamics of molecular machines}

In this study, a molecular machine is viewed in a coarse grained representation as consisting of a set of $N$ spherical particles (beads) $i$ interacting via pairwise potential forces $\boldsymbol{f}_{i j}=f\left(r_{i j}, s\right) \boldsymbol{n}_{i j}$ that depend on the distances $r_{i j}=\left|\boldsymbol{r}_{i j}\right|=\left|\boldsymbol{R}_{i}-\boldsymbol{R}_{j}\right|$ between beads $i$ and $j$ and on the variable $s$ specifying the machine state (see below). Here, $\boldsymbol{n}_{i j}=\boldsymbol{r}_{i j} / r_{i j}$ is the unit vector along the direction connecting particles $i$ and $j$. At low Reynolds numbers characteristic of the microscopic system, inertia is absent and the bead velocities are directly determined by the applied forces. In the simplest approximation, the velocity $\boldsymbol{v}_{i}=\mu \boldsymbol{F}_{i}$ of each bead depends only on the total force $\boldsymbol{F}_{i}=\sum_{j} \boldsymbol{f}_{i j}$ applied to this particle and on its mobility coefficient $\mu$.

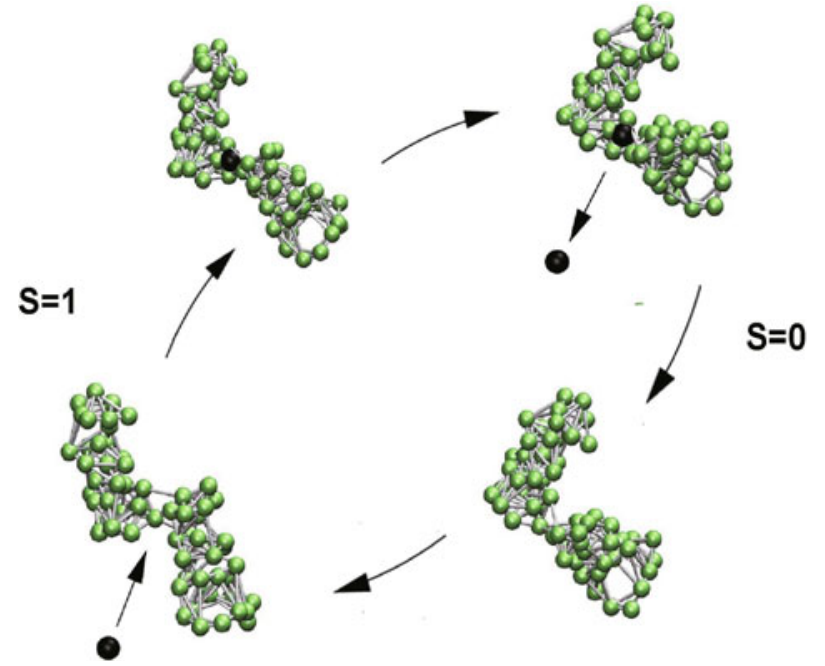

Fig. 1. (Color online) Conformational cycle of a model molecular machine consisting of a network of linked beads. Upon ligand binding to the stable open conformation (lower left) a series of conformational changes take place that lead to the new stable closed conformation (top right). The variable $s=1$ along this path. Release of product from the closed state (signaled by the change to $s=0$ ) leads to conformational changes that return the system to the open state.

When the energy is supplied, a machine, otherwise fluctuating around its equilibrium conformation (specified by the equilibrium distance between beads $\left.\left\{r_{i j}^{(0)}(s=0)\right\}\right)$, makes a conformational cycle. This cycle is described as a sequence of two different relaxation processes, which are specified by a binary variable $s$. The time evolution of the bead positions obeys equations

$$
\begin{aligned}
\frac{d \boldsymbol{R}_{i}}{d t} & =-\mu \frac{\partial \mathcal{F}\left(\left\{\boldsymbol{R}_{i}\right\} ; s\right)}{\partial \boldsymbol{R}_{i}} \\
& =\mu \sum_{j}\left\{f_{0}\left(r_{i j}\right)+s \Delta f\left(r_{i j}\right)\right\} \boldsymbol{n}_{i j},
\end{aligned}
$$

where $\mathcal{F}$ is the free energy of the machine and the value of $s$ depends on the machine internal state, i.e., energetically activated $(s=1)$ or not $(s=0)$. The second equality shows the decomposition of the magnitude of the pair force $f\left(r_{i j}\right)$ into an equilibrium (inactive) part, $f_{0}\left(r_{i j}\right)$, and the force generated due to the activation, $\Delta f\left(r_{i j}\right)$.

We illustrate the machine cycle in more detail assuming that chemical energy is supplied by ligand binding (Fig. 1), although other forms of energy input, such as illumination, are also possible. Suppose that a machine in its equilibrium state is characterized by $s=0$. The machine cycle begins when the ligand (such as an ATP molecule) arrives. In our model, this is described by a change of the internal variable $s$ from $s=0$ to $s=1$ upon ligand binding. As a result of this change, the machine is displaced from equilibrium, $\left\{r_{i j}^{(0)}(s=0)\right\}$, and begins relaxation to a new stable configuration, $\left\{r_{i j}^{(0)}(s=1)\right\}$, with the ligand. This relaxation is described by equation (1) with $s=1$. After some time $\tau_{1}$, the perturbed system 
approaches a new equilibrium state. Then, a chemical reaction converting the ligand into a product occurs. For simplicity, we assume that the product is immediately released. Thus, at time $t=\tau_{1}$ the change of the variable $s$ from $s=1$ back to $s=0$ occurs. The conformation in which the molecule finds itself after this transition is however unstable, so that the system performs another conformational relaxation motion, also described by equation (1), but with $s=0$. The relaxation proceeds and at time $t=\tau=\tau_{1}+\tau_{2}$, when the equilibrium conformation is approached, the machine cycle ends. Now, another ligand can bind to the machine, initiating the next cycle. The effects of hydrodynamic interactions on the cyclic dynamics of such machines have been studied [18].

Thus, the operation cycle essentially consists of two relaxational motions which occur in the intervals $0<t<\tau_{1}$ and $\tau_{1}<t<\tau$. The first stage of the conformational motion (with internal state $s=1$ ) starts from the equilibrium state of the free protein and ends near the equilibrium state of the protein-ligand complex. The second stage of the motion (with internal state $s=0$ ) begins with the final conformation reached by the molecule in the first stage and brings the free molecule close to its equilibrium conformation. These two motions generally follow different paths and proceed with different velocities.

In equation (1), interactions among the beads through hydrodynamical flows in the solvent are neglected. In reality, however, such flows induced by the motion of a bead influence the other beads in the system. These hydrodynamic interactions can be taken into account by introducing a conformation-dependent mobility tensor, so that the equations of motion of our system take the form:

$$
\begin{aligned}
\frac{d \boldsymbol{R}_{i}}{d t} & =\sum_{j=1}^{N} \mathcal{L}_{i j}\left(r_{i j}\right) \cdot \boldsymbol{F}_{j} \\
& =\sum_{j, k=1}^{N} \mathcal{L}_{i j}\left(\boldsymbol{r}_{i j}\right)\left\{f_{0}\left(r_{j k}\right)+s \Delta f\left(r_{j k}\right)\right\} \cdot \boldsymbol{n}_{j k} .
\end{aligned}
$$

Our analytical results, which are obtained below, are general and do not depend on the choice of a particular approximation for the mobility tensor. For example, in the far-field Oseen approximation [19], which is valid when distances between the beads are much larger than their sizes, the mobility tensor has components

$$
\mathcal{L}_{i j}(\boldsymbol{r})=\left\{\begin{array}{ll}
\mu \mathcal{I} & (i=j) \\
\mu \frac{3 a}{4 r}\left(\mathcal{I}+\frac{\boldsymbol{r} \otimes \boldsymbol{r}}{r^{2}}\right) & (i \neq j)
\end{array},\right.
$$

where the mobility coefficient is $\mu=1 /(6 \pi \eta a), \eta$ and $a$ are the solvent viscosity and the hydrodynamic radius of a bead, and $\mathcal{I}$ is the $3 \times 3$ unit matrix. (Approximations which are more accurate at short distances, such as the Rotne-Prager tensor used in Sect. 5, are available). Since the beads are assumed to be structureless and spherical, the rotation of each bead is irrelevant and rotational degrees of freedom need not be considered.

\section{Propulsion velocities and stall forces}

Since the forward and backward motions of the cycle of a molecular machine are generally not reciprocal, a freely suspended machine will propel itself through the fluid and also rotate by some angle after each of its cycles. If a machine is bound to some support such as a membrane, it will exert a propulsion force on the support and a stall force is needed to prevent its motion.

\subsection{Propulsion velocity and angular velocity}

The net propulsion velocity $\boldsymbol{V}_{p}$ can be defined as the velocity of the center of mass of the machine, averaged over a single cycle,

$$
\boldsymbol{V}_{p}=\frac{1}{\tau} \int_{0}^{\tau} \boldsymbol{V}(t) d t
$$

where the instantaneous velocity $\boldsymbol{V}(t)=d \boldsymbol{R}(t) / d t$ of the center of mass is

$$
\boldsymbol{V}(t)=\frac{1}{N} \sum_{i} \frac{d \boldsymbol{R}_{i}}{d t}=\frac{1}{N} \sum_{i, j, k=1}^{N} \mathcal{L}_{i j}\left(r_{i j}\right) f\left(r_{j k} ; s(t)\right) \cdot \boldsymbol{n}_{j k}
$$

We assume for simplicity that all beads have the same mass.

Similarly, the net angular velocity $\boldsymbol{\Omega}_{p}$ can be defined as

$$
\Omega_{p}=\frac{1}{\tau} \int_{0}^{\tau} \Omega(t) d t
$$

where the instantaneous angular velocity is

$$
\boldsymbol{\Omega}(t)=\frac{\sum_{i} \Delta \boldsymbol{R}_{i} \times d \boldsymbol{R}_{i} / d t}{\sum_{i} \Delta \boldsymbol{R}_{i}^{2}},
$$

with $\Delta \boldsymbol{R}_{i}(t)=\boldsymbol{R}_{i}(t)-\boldsymbol{R}(t)$, the relative position of each bead with respect to the center of mass.

\subsection{Stall force}

Suppose that one of the beads (with $i=1$ ) is immobilized by applying the stall force $\boldsymbol{F}^{(1)}(t)$. Then, the equations of motion of the beads are

$$
\frac{d \boldsymbol{R}_{i}}{d t}=\sum_{j, k} \mathcal{L}_{i j} f\left(r_{j k} ; s\right) \cdot \boldsymbol{n}_{j k}+\mathcal{L}_{i 1} \cdot \boldsymbol{F}^{(1)} .
$$

From the immobilization condition $d \boldsymbol{R}_{1}(t) / d t=0$, we get an expression for the instantaneous, time-dependent stall force

$$
\begin{aligned}
\boldsymbol{F}^{(1)}(t)= & -\sum_{j, k} \mathcal{L}_{11}^{-1} \mathcal{L}_{1 j} f\left(r_{j k} ; s\right) \cdot \boldsymbol{n}_{j k} \\
= & -\sum_{k} f\left(r_{1 k} ; s\right) \boldsymbol{n}_{1 k} \\
& -\sum_{j(\neq 1), k} \mathcal{L}_{11}^{-1} \mathcal{L}_{1 j} f\left(r_{j k} ; s\right) \cdot \boldsymbol{n}_{j k} .
\end{aligned}
$$


The net stall force $\boldsymbol{F}_{s}$ is defined as the instantaneous stall force $\boldsymbol{F}^{(1)}(t)$ averaged over a single cycle,

$$
\boldsymbol{F}_{s}=\frac{1}{\tau} \int_{0}^{\tau} \boldsymbol{F}^{(1)}(t) d t
$$

By substituting equation (9) into equation (8), we obtain equations of motion for the rest of the system when bead $i=1$ is immobilized,

$$
\frac{d \boldsymbol{R}_{i}}{d t}=\sum_{j, k} \tilde{\mathcal{L}}_{i j} f\left(r_{j k}, s\right) \cdot \boldsymbol{n}_{j k}, \quad(i \neq 1) .
$$

Here the effect of the immobilization appears as a change in the hydrodynamic interactions,

$$
\tilde{\mathcal{L}}_{i j}=\mathcal{L}_{i j}-\mu^{-1} \mathcal{L}_{i 1} \mathcal{L}_{1 j}
$$

Immobilization not only prevents translational motion of the machine but also influences hydrodynamic interactions among its parts.

When hydrodynamic interactions among the machine beads are absent (i.e., the mobility tensor is diagonal), propulsion does not take place. In the absence of hydrodynamic interactions each bead acts independently and the solvent does not detect the presence of a structured asymmetrical object. More specifically, when the mobility tensor is diagonal, equation (5) yields

$$
\boldsymbol{V}(t)=\frac{1}{N} \mu \sum_{i, j} f\left(r_{i j} ; s\right) \boldsymbol{n}_{i j}=0
$$

as a consequence of momentum conservation. Similarly, it can be easily shown that

$$
\begin{aligned}
\boldsymbol{\Omega}(t) \sim & \frac{\mu}{2} \sum_{i k}\left(\left(\Delta \boldsymbol{R}_{i} \times f\left(r_{i k} ; s\right) \boldsymbol{n}_{i k}\right)\right. \\
& -\left(\Delta \boldsymbol{R}_{k} \times f\left(r_{i k} ; s\right) \boldsymbol{n}_{i k}\right)=0,
\end{aligned}
$$

so that the instantaneous angular velocity is also zero due to the conservation of angular momentum.

In the absence of collective hydrodynamic effects, the stall force is equal to the sum of the elastic forces acting on bead $i=1$ from its neighbors,

$$
\boldsymbol{F}^{(1)}(t)=-\sum_{k} f\left(r_{1 k} ; s\right) \boldsymbol{n}_{1 k}
$$

Unlike the propulsion velocity $\boldsymbol{V}(t)$, this instantaneous stall force does not vanish. By substituting equation (8) into equation (5), we obtain the relation between the instantaneous velocity of the center of mass and the stall force:

$$
\begin{aligned}
\boldsymbol{V}(t) & =\frac{1}{N} \mu\left[\sum_{i, j} f\left(r_{i j} ; s\right) \boldsymbol{n}_{i j}+\boldsymbol{F}^{(1)}(t)\right] \\
& =\frac{1}{N} \mu \boldsymbol{F}^{(1)}(t) .
\end{aligned}
$$

In this case, the instantaneous velocity is not zero because of the presence of the external force, and is proportional to the instantaneous stall force. After the time averaging over a cycle, however, the net propulsion vanishes due to the immobilization, i.e., $\boldsymbol{V}_{p}=(1 / \tau) \int_{0}^{\tau} \boldsymbol{V}(t) d t=0$; therefore, the above equation becomes

$$
\boldsymbol{F}_{s}=\frac{N}{\mu} \boldsymbol{V}_{p}=0
$$

and the net stall force also vanishes. This is a coarse grained force balance equation which is valid on time scales longer than the cycle time $\tau$. Thus, both the propulsion velocity and the net stall force vanish when hydrodynamic interactions are neglected.

\section{Weak perturbation cycles}

We next discuss the propulsion properties of a machine which performs a small amplitude conformational cycle. Let us write the positions of the particles as

$$
\boldsymbol{R}_{i}(t)=\boldsymbol{R}_{i}^{(0)}(s=0)+\delta \boldsymbol{R}_{i}(t),
$$

where $\delta \boldsymbol{R}_{i}(t)$ represents small deviations from equilibrium positions. We also denote the vector connecting the equilibrium positions with and without the ligand as

$$
\boldsymbol{\xi}_{i}=\boldsymbol{R}_{i}^{(0)}(s=1)-\boldsymbol{R}_{i}^{(0)}(s=0)
$$

In this section, we suppose that the perturbation corresponding to ligand binding is weak, so that all $\left|\boldsymbol{\xi}_{i}\right|$ are of order $\varepsilon$ where $\varepsilon \ll 1$ is a small parameter. Then, the small deformation responsible for the restoring force in the forward cycle $(s=1)$ is written as

$$
\boldsymbol{R}_{i}(t)-\boldsymbol{R}_{i}^{(0)}(s=1)=\delta \boldsymbol{R}_{i}(t)-\boldsymbol{\xi}_{i}
$$

We assume that that, within each cycle, the forward motion (with $s=1$ ) has duration $\tau_{1}$ and the reverse motion (with $s=0$ ) has duration $\tau_{2}$, so that the total cycle time is $\tau=\tau_{1}+\tau_{2}$.

In a small deformation cycle, we can expand the equations for the net propulsion velocity (Eqs. (4) and (5)) and retain only terms which are of linear order in the deformation. Noting that in this linear regime the mobility tensor $\mathcal{L}$ and the unit vector $\boldsymbol{n}_{i j}$ can be approximated by those in the equilibrium state, the linearized equations of motion become

$$
\frac{d \delta \boldsymbol{R}_{i}}{d t}=\sum_{l} \mathcal{M}^{*}{ }_{i l}\left(\delta \boldsymbol{R}_{l}-s(t) \boldsymbol{\xi}_{l}\right)
$$

where $\mathcal{M}^{*}$ is the super matrix (with components of $3 \times 3$ matrix) with the following form

$$
\mathcal{M}^{*}{ }_{i l}=\left.\sum_{j, k} \mathcal{L}\left(r_{i j}^{(0)}\right) \boldsymbol{n}_{j k}^{(0)}\left(\partial f_{0} / \partial r\right)\right|_{r_{j k}^{(0)}} \boldsymbol{n}_{j k}^{(0)}\left(\delta_{l j}-\delta_{l k}\right) .
$$


By introducing $3 N$ components vectors $\rho \equiv\left(\delta R_{1, x}\right.$, $\left.\delta R_{2, y}, \cdots, \delta R_{N, z}\right)$ and $\chi \equiv\left(\xi_{1, x}, \xi_{2, y}, \cdots, \xi_{N, z}\right)$ equation (21), thus, can be rewritten as

$$
\frac{d \rho}{d t}=\mathcal{M}[\rho-s(t) \chi]
$$

where $3 N \times 3 N$ matrix $\mathcal{M}$ is constructed from the super matrix $\mathcal{M}^{*}$ as $\mathcal{M}_{3(i-1)+\alpha} 3(j-1)+\beta=\left(\mathcal{M}^{*}{ }_{i j}\right)_{\alpha \beta}$. We then diagonalize $\mathcal{M}$ with the matrix $\mathcal{V}$ as

$$
\mathcal{M V}=-\mathcal{V} \Gamma
$$

where $\Gamma_{n n^{\prime}}=\gamma_{n} \delta_{n n^{\prime}}$ is a diagonal matrix. The small deformation can be represented by the sum of individual modes

$$
\rho_{n}(t)=\sum_{n^{\prime}} V_{n n^{\prime}} q_{n^{\prime}}(t)
$$

where it is noted that the modes corresponding to the translation and rotation are not relevant here. Putting equations $(23)-(25)$ together, we arrive at the following normal mode equation

$$
\frac{d q_{n}(t ; s)}{d t}=-\gamma_{n}\left[q_{n}(t ; s)-s(t) q_{n}^{(0)}\right]
$$

where $\boldsymbol{q}^{(0)}$ is defined by

$$
\chi_{n}=\sum_{n^{\prime}} V_{n n^{\prime}} q_{n^{\prime}}^{(0)} .
$$

The solution is

$$
\begin{aligned}
& q_{n}(t ; s)= \\
& \begin{cases}q_{n}^{(0)}\left(1-e^{-\gamma_{n} t}\right) & \left(s=1 \Leftrightarrow 0 \leq t \leq \tau_{1}\right) \\
q_{n}\left(\tau_{1} ; s=1\right) e^{-\gamma_{n}\left(t-\tau_{1}\right)} & \left(s=0 \Leftrightarrow \tau_{1} \leq t \leq \tau\right) .\end{cases}
\end{aligned}
$$

Here, we have assumed that the duration $\tau_{2}=\tau-\tau_{1}$ of the reverse motion is long enough for the full relaxation to the initial state to take place. This condition is satisfied if $\gamma_{\min } \tau_{2} \gg 1$, where $\gamma_{\min }$ is the relaxation rate constant of the slowest normal mode. Each mode independently contributes to the propulsion, so we get for the $\alpha$ component of the propulsion velocity

$$
\begin{aligned}
\left(V_{p}\right)_{\alpha}= & \frac{1}{\tau N} \sum_{i=1}^{N} \sum_{n, n^{\prime}=1}^{3 N-6} M_{3(i-1)+\alpha} V_{n n^{\prime}} \\
& \times \int_{0}^{\tau} d t\left[q_{n^{\prime}}(t)-s(t) q_{n^{\prime}}^{(0)}\right] .
\end{aligned}
$$

By denoting the $n^{\prime}$-dependent coefficient in the first line (divided by $N$ ) as $C_{n^{\prime}}^{(\alpha)}$, we finally obtain

$$
\begin{aligned}
\left(V_{p}\right)_{\alpha} & =\sum_{n^{\prime}} C_{n^{\prime}}^{(\alpha)} \frac{1}{\tau} \int_{0}^{\tau} d t\left[q_{n^{\prime}}(t ; s)-s(t) q_{n^{\prime}}^{(0)}\right] \\
& =-\sum_{n^{\prime}} C_{n^{\prime}}^{(\alpha)} q_{n^{\prime}}^{(0)}\left(1-e^{-\gamma_{n^{\prime}} \tau_{1}}\right) \gamma_{n^{\prime}}^{-1} e^{-\gamma_{n^{\prime}} \tau_{2}} .
\end{aligned}
$$

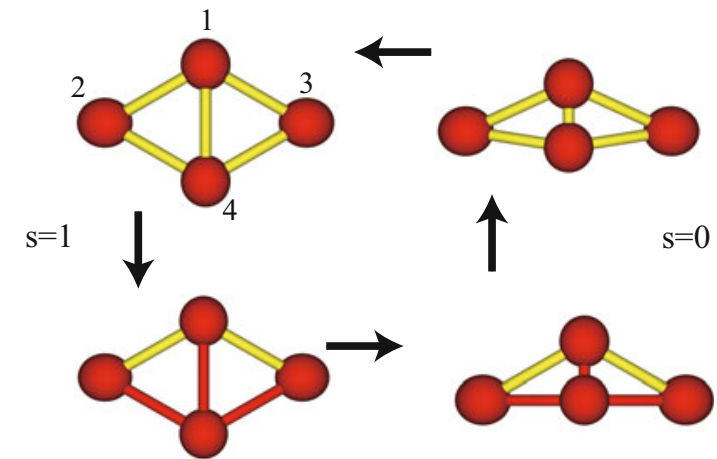

Fig. 2. (Color online) Cycle of the simple model machine. The energetic activation leads to the change of the interaction parameters (natural link lengths) for the lower three beads (2, 3 and 4). This gives rise to a change in the shape of the object. When this change has taken place, the structural perturbation is removed and the object relaxes back to its equilibrium shape. Note that the forward and backward motions are not the same because the respective interaction parameters are different.

Because $\gamma_{\min } \tau_{2} \gg 1$, the net propulsion velocity is vanishingly small. Since the stall force is related to the propulsion velocity with the proportional (friction) constant which is independent of $\varepsilon$ in the weak perturbation cycle, the net stall force is also exponentially weak in this limit.

Thus, these calculations show that the propulsion effects are exponentially small in the linear response approximation. For small cycles with $\varepsilon \ll 1$, the expansions of the propulsion velocity and the net stall force must begin with quadratic terms of order $\varepsilon^{2}$.

\section{Application to a simple molecular machine}

To demonstrate how our general results can be applied to the analysis of propulsion properties of a particular machine, we consider in this section a simple example of a machine with only four particles. The machine consists of four beads with diameter $d=2 a$, which are connected through elastic links to form the object shown in Figure 2. The elastic energy of the machine is

$$
\mathcal{F}=\frac{1}{2} \sum_{i \neq j} k_{i j}\left(r_{i j}-r_{i j}^{(0)}(s)\right)^{2} .
$$

There is no link between particles 2 and $3\left(k_{23}=0\right)$ and all other elastic constants are assumed to be equal $\left(k_{i j}=k\right)$. At equilibrium $(s=0)$, all links have the same length $r_{i j}^{(0)}(s=0)=2 d$. The ligand binding is implicitly modeled as an energetic activation, which modifies the interactions between bead 4 and other three beads. In the energetically activated state $(s=1)$, interactions between the beads are changed, so that $r_{14}^{(0)}(s=1)=(2-\varepsilon) d$ and $r_{24}^{(0)}(s=1)=r_{34}^{(0)}(s=1)=\sqrt{\left(\varepsilon^{2}-2 \varepsilon+4\right)} d$, where $\varepsilon$ gauges the magnitude of the structural perturbation. The machine cycle consists of a sequence of two relaxation motions (see Fig. 2). The cycle begins with the energetic activation, i.e. a transition from $s=0$ to $s=1$. This is 
$\Delta \mathrm{R}(\mathrm{t}) / \mathrm{d}$

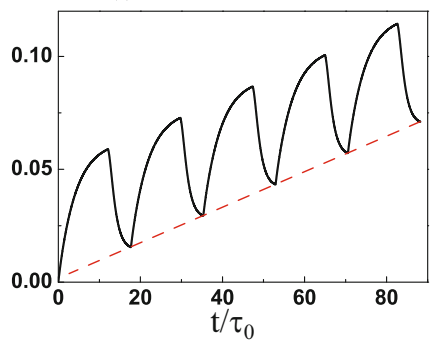

$\mathrm{F}^{(1)}(\mathrm{t}) / \mathrm{kd}$

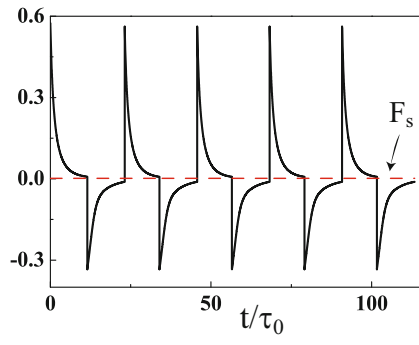

Fig. 3. (Color online) (left) Displacement of the center of mass and (right) the instantaneous stall force $F^{(1)}$ as functions of time with $\varepsilon=d$. Dotted lines indicate mean values.

followed by relaxation of the activated object towards its new equilibrium shape. The elastic energy decreases and, at some time $\tau_{1}$, it becomes smaller than $\mathcal{F}_{\text {min }}$. At this moment, the reverse transition from $s=1$ to $s=0$ occurs and the energy of the object is increased. Therefore, relaxation to the equilibrium state of the non-activated object begins now. The cycle ends when the elastic energy becomes again smaller than $\mathcal{F}_{\text {min }}$. The condition $\mathcal{F}=\mathcal{F}_{\text {min }}$ determines durations $\tau_{1}$ and $\tau_{2}$ of the activated and nonactivated parts of cycle. The total cycle time is $\tau=\tau_{1}+\tau_{2}$. In our simulations, $\mathcal{F}_{\text {min }}=10^{-4} k d$.

To determine internal motions of the model machine in the presence of hydrodynamic interactions, we have numerically integrated equation (2) with the Rotne-Prager mobility tensor,

$\mathcal{L}_{i j}(\boldsymbol{r})=\left\{\begin{array}{ll}\mu \mathcal{I} & (i=j) \\ \mu \frac{3 a}{4 r}\left[\left(1+\frac{2 a^{2}}{3 r^{2}}\right) \mathcal{I}+\left(1-\frac{2 a^{2}}{r^{2}}\right) \frac{\boldsymbol{r} \otimes \boldsymbol{r}}{r^{2}}\right] & (i \neq j)\end{array}\right.$,

which differs from the Oseen mobility tensor (3) in that the next-higher order term is taken into account in the farfield approximation. In numerical simulations, distances are scaled by $a$ and time by $\tau_{0}=(\mu k)^{-1}$.

Figure 3 (left) shows the propulsion of this model machine. Its center of motion moves along the symmetry direction. The machine is first propelled upward by $\Delta R / d \simeq 0.06 d$ after the transition from $s=0$ to $s=1$, i.e., after the energetic activation. In the subsequent backward motion after the transition from $s=1$ to $s=0$, the original shape of the machine is recovered. However, the center of mass of the machine does not return to its initial position after the completion of the cycle. The net propulsion velocity can be calculated from the slope in Figure 3 (left) or from the analytical expressions given by equations (4) and (5). The result is $V_{p}=7.88 \times 10^{-4} d / \tau_{0}$ with the magnitude of the structural perturbation $\varepsilon=d$.

Numerical simulations of the immobilized model machine have also been performed. To prevent translational motion, bead 1 was fixed. The stall force, which must be applied at this bead to stop the motion, was monitored. Figure 3 (right) shows the time dependence of the stall force and its time average. The results of such direct evaluation yield a net stall force of $F_{s}=1.89 \times 10^{-3} k d$ with $\varepsilon=d$. This result has also been obtained directly using the analytical expressions in equations (9) and (10).

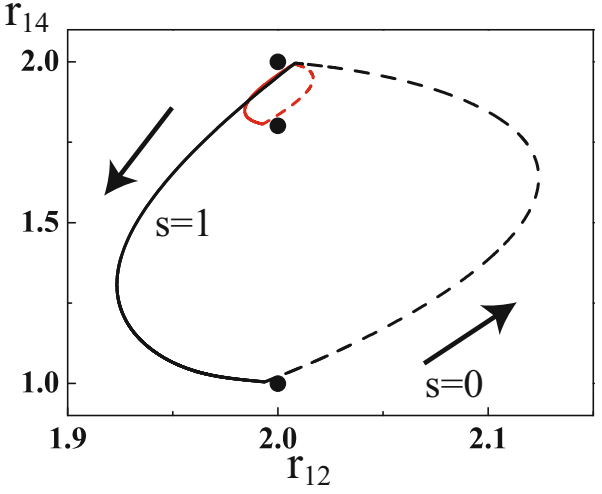

Fig. 4. (Color online) Projections of the cyclic trajectory on the plane of distances between particles 1 and 4 and between 1 and 2. Two cycles, corresponding to weak $(\varepsilon=0.2 d)$ and strong $(\varepsilon=d)$ structural perturbations, are shown. The forward motions are shown by solid lines, whereas the backward motions are displayed by dashed lines.
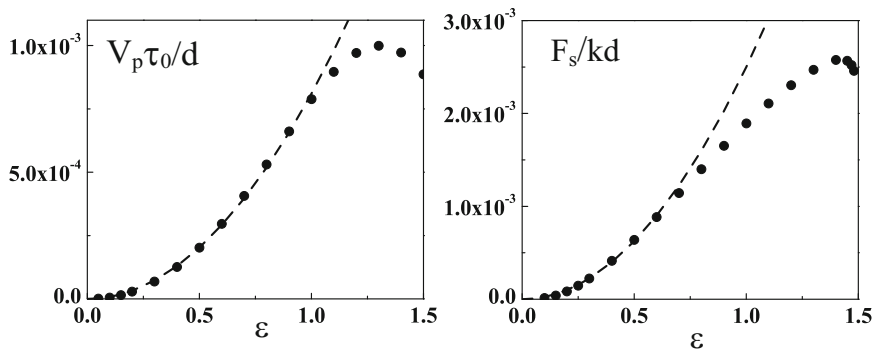

Fig. 5. Net propulsion velocity (left) and net stall force (right) as functions of the degree of the strength $\varepsilon$. Dashed lines show the quadratic fits.

Depending on the magnitude $\varepsilon$ of the structural perturbation, the cycles of the model machine can be large or small. Figure 4 shows examples of the trajectories corresponding to such cycles. Solid lines in this figure correspond to forward motion with $s=1$ and the dashed lines to the backward motion with $s=0$. The forward and backward trajectories are always different, but they approach each other for the small cycle.

In Figure 5, the computed values of the net propulsion velocity (left) and the net stall force (right) for different magnitudes $\varepsilon$ of structural perturbations are given. For comparison, we also show here the quadratic fit (dashed curves). For weak perturbations, these properties are indeed proportional to $\varepsilon^{2}$, in agreement with our analysis in Section 4.

\section{Conclusions}

Molecular machines perform conformational cyclic motions when chemical energy is supplied. Because cyclic trajectories are generally non-reciprocal, these machines can propel themselves through the fluid in which they are immersed. We have modeled the conformational cycles of machines by introducing ligand binding as a structural perturbation and obtained analytical estimates for the 
propulsion velocity and the stall force needed to immobilize a machine. We have further generally demonstrated that the propulsion effects vanish in the kinetic linear response regime and, therefore, the nonlinearity of relaxation processes is important for hydrodynamic propulsion. Simulations of the cyclic dynamics of a simple model molecular machine were used to illustrate the results.

While the analysis presented in this paper focused on general aspects of the phenomena and the construction of a framework in which to describe molecular machine propulsion, applications to actual protein machines can be carried out using the results of this paper. As noted earlier, almost all protein machines execute conformational cycles that are generally non-reciprocal. In our analysis, conformational dynamics within the cycle was taken as known. The progress in dynamical modeling of active biopolymers already allows one to determine conformational motions within the entire cycle for relatively small model machines $[18,20]$. If the conformational dynamics in the entire cycles of real molecular machines is known, the results of our study can be used to investigate their propulsion effects.

\section{References}

1. B. Alberts, Cell 92, 291 (1998)

2. B.K. Kinbara, T. Aida, Chem. Rev. 105, 1377 (2005)
3. R.D. Astumian, Science 276, 917 (1997)

4. F. Jülicher, A. Ajdari, J. Prost, Rev. Mod. Phys. 69, 1269 (1997)

5. P. Ball, Nanotechnology 13, R15 (2002)

6. E.R. Kai, D.A. Leigh, F. Zerbetto, Angew. Chem. Int. Ed. 46, 72 (2007)

7. E.M. Purcell, Am. J. Phys. 45, 3 (1977)

8. For a more detailed analysis see, A. Shapere, F. Wilczek, Phys. Rev. Lett. 58, 2051 (2003)

9. L.E. Becker, S.A. Koehler, H.A. Stone, J. Fluid Mech. 490, 15 (2003)

10. D. Tam, A.E. Hosoi, Phys. Rev. Lett. 98, 068105 (2007)

11. A. Najafi, R. Golestanian, Phys. Rev. E 69, 062901 (2004)

12. C.M. Pooley, G.P. Alexander, J.M. Yeomans, Phys. Rev. Lett. 99, 228103 (2007)

13. D.J. Earl, C.M. Pooley, J.F. Ryder, I. Bredberg, J.M. Yeomans, J. Chem. Phys. 126, 064703 (2007)

14. R. Golestanian, A. Ajdari, Phys. Rev. Lett. 100, 038101 (2008)

15. R. Golestanian, A. Ajdari, Phys. Rev. E 77, 036308 (2008)

16. M. Iima, A.S. Mikhailov, Europhys. Lett. 85, 4401 (2009)

17. R. Golestanian, A. Ajdari, J. Phys.: Cond. Matter 21, 204104 (2009)

18. A. Cressman, Y. Togashi, A.S. Mikhailov, R. Kapral, Phys. Rev. E 77, 050901(R) (2008)

19. J.K.G. Dhont, An Introduction to Dynamics of Colloids (Elsevier, Amsterdam, 1996)

20. Y. Togashi, A.S. Mikhailov, Proc. Natl. Acad. Sci. USA 104, 8697 (2007) 